\title{
Grenzerfahrungen \\ Über Orientierung in einer digitalisierten Welt und den Wert der Sozialen Arbeit
}

PFARRER CHRISTIAN DOPHEIDE ist theologischer Vorstand der Evangelischen Stiftung Hephata, Vorstandsvorsitzender des Verbands diakonischer Dienstgeber in Deutschland (VdDD) und Autor des Buches Zur Digitalisierung des Sozialen (Nomos 2017). www.hephata-mg.de www.v3d.de

\author{
Mit der zunehmenden digitalen und globalen Vernetzung \\ verschieben sich Grenzen - politisch, wirtschaftlich \\ und sozial. Wir befinden uns in einer Übergangsphase, \\ in der das Prinzip der Abgrenzung und das Prinzip der \\ Verknüpfung um Vorherrschaft zu ringen scheinen - \\ bevor sie sich (hoffentlich) in ein gesundes Gleichgewicht \\ einpendeln. Die Folge ist eine flirrende Schwebe, in der \\ wir uns von Grenzen mehr Sicherheit erhoffen, als sie \\ uns noch gewähren könnten, und von Verknüpfungen \\ mehr Tragfähigkeit, als diese bereits bieten.
}

Wir sind geneigt, das Phänomen der Grenze für natürlich zu halten. Schließlich wissen wir, dass unsere Lebenszeit begrenzt ist und auch unsere Gestaltungkraft Grenzen hat. Wir sprechen von natürlichen Grenzen und denken dabei an Gebirgszüge und Flussläufe. Natürliche Grenzen aber sind immer Zonen des fließenden Übergangs. Man kann keinen Pflock einschlagen da, wo ein Berg beginnt oder da, wo Festland ins Meer übergeht. Man kann aber sehr wohl einen Pflock einschlagen da, wo der Geltungsbereich des Grundgesetzes endet und der Geltungsbereich einer anderen Verfassung beginnt. Die Grenze als Demarkationslinie ist eine menschliche Erfindung aus der Jungsteinzeit. Dem Jäger und Sammler war die Grenze seines Reviers noch eine Zone des Übergangs, nämlich des nachlassenden Interesses. Dem Ackerbauern aber ist die Einfriedung seines Feldes eine Zone des höchsten Interesses. Ist nämlich die Wildsau erst einmal durch den Zaun hindurch, dann ist die Ernte hin und es droht der Hungertod.

Seitdem (und damit seit rund 12.000 Jahren) haben wir praktisch alle gesellschaftlichen Arrangements auf die Logik der Grenze als Demarkationslinie, auf die Logik der eindeutigen Unterscheidung abgestellt. Wir haben definiert, welches Kind ehelich ist und welches nicht. Wer Staatsbürger ist und wer nicht. Was Erwerbsarbeit ist und was nicht. Wo das Marktgeschehen beginnt und wo es endet. Eigentlich wissen wir, wie uneindeutig all solche Unterscheidungen sind. Natürlich waren nie alle ehelichen Kinder auch die Kinder einer Ehe. Natürlich lebten nie alle Deutschen in Deutschland noch waren jemals alle Deutschen deutsch. Natürlich war der Übergang zwischen Nachbarschaftshilfe und Erwerbsarbeit immer schon fließend und anthrazitgrau. Natürlich herrschte im Bereich Sozialer Arbeit immer schon Wettbewerb und haben Märkte immer auch eine ganz bedeutende soziale Funktion. Zwecks Strukturierung und Orientierung aber haben sich menschliche Gesellschaften seit 12.000 Jahren angewöhnt, auch Uneindeutiges durch die Konstruktion eindeutiger Unterscheidungen voneinander zu separieren und dadurch handhabbar zu machen.

Es ist ein Treppenwitz der Weltgeschichte, dass ausgerechnet die Digitalisierung, also die Reduktion aller Information auf die binäre Unterscheidung von Einsen und Nullen, dazu führt, dass wir in unseren gesellschaftlichen Arrangements mit eindeutigen Unterscheidungen immer weniger zurechtkommen. Freilich ist der Clou nicht schon die 
Zerlegung von Informationen in eine Folge von Einsen und Nullen. Der Clou ist deren anschließende weltweite Verknüpfung in Echtzeit durch Rechenmaschinen mit rasender Geschwindigkeit.
Ob es nun in dieser flirrenden Schwebe vorwärts gehen muss oder lieber doch wieder zurück, das ist eine Frage, die derzeit weltweit verhandelt wird. Und paradoxerweise sind zurzeit die Rück-

\section{"Es ist ein Treppenwitz der Weltgeschichte, dass ausgerechnet die Digitalisierung, also die Reduktion aller Information auf die binäre Unterscheidung von Einsen und Nullen, dazu führt, dass wir in unseren gesellschaftlichen Arrangements mit eindeutigen Unterscheidungen immer weniger zurechtkommen"}

Insofern ist 1989 das Jahr der Wende nicht wegen des Mauerfalls, sondern wegen Tim Berners Lee, der zeitgleich beim Forschungszentrum CERN mit dem "world wide web « das technische Verfahren zur globalen Verknüpfung der Daten und Dinge entwickelte.

Die Technik der globalen und nahezu verzögerungsfreien Verknüpfung von Daten, Dingen (und nicht zuletzt von Menschen) mindert die Definitionskraft künstlicher Demarkationslinien enorm. Dem Prinzip der Unterscheidung stellt sich nunmehr, mit dynamisch zunehmendem Gewicht, das Prinzip der Verknüpfung zur Seite. Eindeutigkeiten, die zwar meist nur konstruiert waren, aber gerade als solche einer Gesellschaft Orientierung gaben, sind immer schwerer zu halten. Und während Grenzen vornehmlich dazu dienen, Herrschaft zu etablieren, sind Verknüpfungen bestens dazu geeignet, Strukturen der Herrschaft sowohl zu unterlaufen als auch zu überspielen. So geraten wir mit der zunehmenden digitalen und globalen Verknüpfung in eine Übergangsphase, in welcher sich das Prinzip der Abgrenzung und das Prinzip der Verknüpfung mehr und mehr die Waage halten. Eine flirrende Schwebe ist die Folge, in der wir uns von Grenzen mehr Sicherheit erhoffen, als sie uns noch gewähren könnten, sowie von Verknüpfungen mehr Tragfähigkeit, als diese bereits bieten. wärtsgewandten auf dem Vormarsch. Dabei ist die Antwort eigentlich sonnenklar: es muss voran gehen. Und es geht auch voran. Die Welt ist nämlich sehr viel besser, als die meisten von ihr denken. Der kürzlich verstorbene schwedische Mediziner Hans Rosling zeigt dies mit erfrischender Klarheit - neuerdings in einem Buch, das von seinem Sohn und seiner Schwiegertochter posthum herausgebracht wurde. ${ }^{1}$ Seit 200 Jahren geht es aufwärts in der Welt. Und zwar mit enormer Geschwindigkeit. Es geht dramatisch vielen Menschen auf der Welt dramatisch viel besser, als es noch ihren Eltern und Großeltern gegangen ist. Allerdings geht diese rasante Entwicklung einher mit großer Verunsi- die hier besonders betroffen sind: die Wirtschaftsordnung, der Politikbetrieb sowie die Bedingungen Sozialer Arbeit.

\section{In der global vernetzten Welt stellt sich die soziale Frage neu}

Sowohl die Europäische Union im Vertrag von Lissabon als auch die Bundesrepublik Deutschland im Einigungsvertrag von 1990 haben sich bezüglich der geltenden Wirtschaftsordnung auf das Leitbild einer Sozialen Marktwirtschaft festgelegt. Die Kontroversen, welche der Bandbreite dessen geschuldet sind, was mit einer "Sozialen Marktwirtschaft « eigentlich alles gemeint sein kann, müssen an dieser Stelle gar nicht entschieden werden. Hier ist erst einmal nur von Interesse, dass dem Staat im Konzept der Sozialen Marktwirtschaft zwar eine durchaus bedeutsame Rolle zugeschrieben wird, er als eigenes Phänomen aber gar nicht in den Blick kommt. Er ist einfach da. Aus dem Kontext des Marktgeschehens blickend, gilt der Staat als jene Sphäre, welche den Markt umschließt und ihn regelt. Das ist auch, cum grano salis, so gewesen, als die Begriffe »Nationalökonomie « und "Volkswirtschaft " das Licht der Welt erblickten. Der Markt war die Summe aller Tauschvorgänge, die sich innerhalb eines Nationalstaates oder eben eines Volkes vollzogen. Die Nationalstaaten wiederum waren über Im- und Exporte miteinander verkoppelt, welche dann den Weltmarkt darstellten. So in etwa schaut auch bis heute das Weltbild Donald Trumps und seiner Freunde aus. Es hat aber dieses Weltbild mit der Wirklichkeit einer digital und deshalb global vernetzten Ökonomie nur noch wenig zu tun.

\section{"Die Menschheit taumelt nicht ihrem Untergang entgegen. Ihr wird vielmehr im Aufstieg schwindelig"}

cherung - insbesondere für jene, die in den letzten Jahrzehnten leidlich sicher und gut leben konnten. Trotzdem: die Menschheit taumelt nicht ihrem Untergang entgegen. Ihr wird vielmehr im Aufstieg schwindelig. Im Folgenden seien in aller Kürze ${ }^{2}$ drei Felder benannt,
Aufgaben, die zuvor relativ einheitlich beim Nationalstaat zusammenliefen, verteilen sich heute auf staatliche und überstaatliche Ebenen. Ordnungsfunktionen werden zudem mehr und mehr durch multilaterale Vertragswerke unter Gleichrangigen und damit durch 
Netzwerkstrukturen, also durch das Prinzip der Verknüpfung, wahrgenommen. Das haben manche noch nicht verstanden.

Sozialpolitiker der alten Schule etwa sehen sich meist angewiesen auf den Nationalstaat als Ordnungsrahmen sowie als Ebene, über welche Transferleistungen $\mathrm{zu}$ organisieren sind. In keinem zweiten Sektor wird deshalb noch so konsequent nationalstaatlich und nationalökonomisch gedacht und argumentiert wie ausgerechnet im Sektor der Sozialpolitik. Dies führt in eine durchaus tragische Situation, denn in der operativen Praxis legt sich Sozialpolitik alter Schule damit fest auf ein Modell, das sie zum ersten, angesichts der universellen Gültigkeit des Menschenrechts, normativ bereits hinter sich gelassen hat. Das sich aber, zum zweiten, auch praktisch gar nicht mehr restaurieren lässt.

Für die richtigen Ziele der Sozialen Marktwirtschaft braucht es neue, alternative Konzepte, die versuchen, das Prinzip der Verknüpfung zielführend zu nutzen. Alternativen könnten sich bieten durch die Arbeit an einem Netzwerk internationaler, sanktionsbewehrter Vertragsbeziehungen. Vor diesem Hintergrund mag es berechtigte Kritik geben an dem einen oder anderen Freihandelsabkommen. Deren grundsätzliche Ablehnung aber ist unethisch. Jedenfalls hat die Anti-TTIP-Bewegung auch dem Merkantilisten Donald Trump ganz trefflich in die Karten gespielt.

Mit der digitalen und deshalb globalen Verknüpfung der Wirtschaftsprozesse stellt sich die Soziale Frage zwingend global - die Migrationsbewegungen der Gegenwart belegen das. nicht nur zum wirtschaftlichen Aufstieg der deutschen Länder und später Deutschlands, sondern, ineins damit, zu einem ungeheuren Migrationsdruck auf die boomenden Industriezentren und in der Folge zu ganz erheblichen sozialen Verwerfungen. MünchenGladbach, das »rheinische Manchester«, war diesbezüglich ein Hotspot. Hier entstand der »Volksverein für das katholische Deutschland «, historischer Quellgrund christlich-sozialer Politik. Hier kam es zugleich durch das protestantisch geprägte, sozial verantwortliche Unternehmertum zur Gründung der Ev. Stiftung Hephata. Es führten aber bekanntlich erst die Sozialreformen Bismarcks dazu, dass der revolutionäre Druck im Kessel nachließ. Dass es dann 1914 doch noch zur Explosion kam, das ist gerade nicht dem (damals noch analogen) Globalisierungsschub des 19. Jahrhunderts geschuldet, sondern vielmehr dessen Ausbremsung durch den zunehmenden Nationalismus und Protektionismus, der auf der Schwelle zum 20. Jahrhundert immer weiter um sich griff.

\section{Kulturelle Irritationen}

Globale digitale Vernetzung sorgt für globale Interdependenz. Das ist eine schlechte Nachricht, soweit wir gehofft haben auf die Handlungsfähigkeit der Regierung, die wir gewählt haben. Das ist aber eine ziemlich gute Nachricht, soweit wir existentiell betroffen sind von Entscheidungen irgendwelcher Regenten auf der Welt, die wir uns nicht aussuchen konnten. Die weltweite gegenseitige Abhängigkeit durch die digitalisierte Globalisierung ist damit das bedeutendste

\section{"Mit der digitalen und deshalb globalen Verknüpfung der Wirtschaftsprozesse stellt sich die Soziale Frage zwingend globalu}

Vergleichbare Kräfte wirkten, als sich die deutschen Länder zu einem gemeinsamen Wirtschaftsraum verknüpften. Die preußischen Agrarreformen und nachfolgend die schrittweise Errichtung eines deutschen Binnenmarktes führten friedenssichernde Phänomen, das der Menschheit je begegnet ist. Friedenssicherung durch gegenseitige Abhängigkeit ist der Friedenssicherung durch gegenseitige Abschreckung ethisch überlegen. Sie ist auch ökonomisch überlegen, weil auf diese Weise Wohlstand nicht nur nicht verzehrt, sondern vielmehr: vermehrt wird.

Die Mehrfachbindung einer demokratisch gewählten Regierung an sowohl inländische als auch globale Einflussgrößen führt allerdings Regierung und Regierte in eine ganz erhebliche Irritation. Zur Demokratie gehört nach Meinung vieler die Idee, um nicht zu sagen: die Ideologie der ausschließlichen Bindung der Regierung an den Willen der Regierten. So einfach ist das aber nicht. Es muss auch die Demokratie, will sie Zukunft haben, ihre Rechnung mit den Wirten machen, bei denen sie anschreiben lässt.

Die Prävalenz externer Faktoren ist typisch für die Steuerungslogik von Unternehmen. Unternehmensführung gelingt nur in dem Maße, in dem sich interne Formen der Mitbestimmung den externen Anforderungen unterordnen. Der Einzug solcher Logik ins demokratische Geschehen macht aber etwas mit dem Wähler. Seine eigentlich eindeutige Rolle als Staatsbürger wird doppeldeutig. Vor der Wahl erscheint er dem Politikbetrieb wie ein Kunde, den man mit einer Reihe von Qualitätsversprechen zur gewünschten Kaufentscheidung bewegen muss. Das beliebt gewordene Bonmot, die Politik müsse »liefern «, illustriert dieses Rollenspiel sehr präzise. Nach der Wahl aber fühlt sich der Bürger nicht mehr wie König Kunde, sondern wie ein Mitarbeiter, der mit den Entscheidungen aus der Vorstandsetage irgendwie zurechtkommen muss. Es ist aber der Staatsbürger weder Kunde noch Mitarbeiter. Und es ist »die Politik« weder die »Deutschland AG « noch eine Lieferando-Plattform.

Bleiben Wahlkämpfer und Wähler bei ihren alten Mustern, dann entstehen jene Rituale, die sich demokratiegefährdend auswirken können. Wahlversprechen werden gegeben, können aber gar nicht gehalten werden. Und ganz gleich, wen man wählt: alle handeln, sind sie erst einmal im Amt, mehr oder weniger gleich. Wie bei einer Übersprungshandlung werden dann gern alternative Politikfelder besetzt, um Handlungsfähigkeit zu simulieren. Besonders beliebt sind dabei diverse Schutzlücken im Straf- und Verkehrsrecht sowie die Bekämpfung von allerlei Süchten. Zudem eignen sich die mit der digitalen, globalen Vernetzung 
einhergehenden kulturellen Irritationen bestens dazu, ideologisch aufgeladen zu werden und dann Politikfelder herzugeben, auf denen populistisch geprägte Regierungen ihre Handlungsfähigkeit simulieren.

Dieser populistische Politikstil lässt sich als regressive Reaktion bezeichnen. Reaktionär ist er, weil er auf die digitale Revolution bloß reagiert, aber keine einzige konstruktive Idee zu ihrer Gestaltung vorhält. Regressiv ist er, weil er stattdessen in kindlicher Manier die Illusion vermittelt, alles würde wieder
Demokratie in vernetzter Zeit nicht mehr.

\section{Die Erfahrung als Mensch bedeutsam zu sein}

Seit ihren Anfängen erlebt die Menschheit, dank Arbeitsteilung, eine beständige Steigerung ihrer Produktivität und damit eine Besserung ihrer Lebensbedingungen. Arbeitsteilung aber ist nicht nur ein ökonomisches, sondern zugleich ein soziales Phänomen. Sie ist ein Kommunikationsgeschehen. Wer

\section{"Demokratische Wahlen unter}

\section{vernetzten Bedingungen können keine 'Richtungsentscheidungs im alten Sinne mehr sein«}

gut, wenn man nur auf der Zeitleiste weit genug zurück krabbelt und sich alter Lösungsmuster bedient. Für diese Art reaktionärer und zugleich regressiver Politik ist es übrigens völlig gleich, ob sie sich als »links « oder als »rechts « stilisiert, denn sie läuft immer auf dasselbe hinaus: Simulation von Lösungen statt simultaner Lösung von vernetzten Problemsträngen.

Demokratische Wahlen unter vernetzten Bedingungen können keine »Richtungsentscheidung « im alten Sinne mehr darstellen. In Wahlen wird vielmehr die Vertrauensfrage gestellt und beantwortet. Es gewinnt das Personaltableau, welches der Mehrheit der Wahlberechtigten Dreierlei vermitteln konnte:

- Wir verstehen die Komplexitäten der Gegenwart und wir sind in der Lage, diese Komplexität in einfacher, bildhafter Sprache zu erläutern.

- Wir sind bereit und in der Lage, die Interessen aller im Lande lebenden Menschen im Kontext weltweiter Abhängigkeiten geduldig und beharrlich zu vertreten.

- Uns ist unbedingt daran gelegen, bei der unvermeidlichen Reduktion aller Wünsche auf das Maß des Machbaren Fairness walten zu lassen.

Welterklärung, Interessensvertretung, Fairness: das ist ein wahrhaft olympischer Dreisprung. Einfacher aber geht aus diesem Kommunikationsgeschehen ausgegrenzt bleibt, nimmt Schaden an seiner Seele selbst dann, wenn ihm der Lebensbedarf materiell gedeckt wird. Vor diesem Hintergrund geht eine Verengung der Sozialpolitik auf Fragen der Umverteilung am Kern des Problems vorbei. Umverteilung, soweit man sich auf sie beschränkt, reduziert das Thema der sozialen Gerechtigkeit auf die Frage nach der angemessenen Kaufkraft. Damit aber reduziert sie Menschen auf ihre Rolle als Kunden. Jeder Mensch will sich aber auch als Lieferant erleben. Und wer um Lieferung gar nicht erst gebeten wird, der liefert ungefragt. Und zwar das, was niemand braucht und niemand will. Es gehört zu den
Fähigkeit, wie man derzeit vielerorts beobachten kann.

Optimisten der Digitalwirtschaft widersprechen der Befürchtung, es ginge uns mit dem Internet der Dinge allmählich die Arbeit aus. Es sei schon immer so gewesen, dass der ökonomische Fortschritt viel Arbeit eliminiert und zugleich viel neue Arbeit hervorgebracht hat. Und es ist ja wahr: Früher brauchte es keine Datenschutzbeauftragten, heute braucht es sie. Insofern könnten die Digitaloptimisten recht haben. Allerdings nur dann, wenn in diese Betrachtung das große Feld der Sozialen Arbeit einbezogen wird.

Soziale Dienstleistungen werden meist mit sozialen Transferleistungen in einen Topf geworfen. Es lohnt sich aber, zwischen beiden Kategorien klar zu unterschieden. Natürlich braucht es soziale Transferleistungen zur Stärkung der Kaufkraft Benachteiligter und man muss darüber streiten, wie sie zur Fairness beitragen können. Wahrscheinlich gibt es ihrer viel zu viele. Die wenigsten wirken ja von »oben « nach »unten«. Das Gros wird vielmehr in einem fröhlichen, wahlkampfgetriebenen Ringelspiel im Kreis herumgereicht zwischen ganz unterschiedlichen Anspruchsgruppen ein und derselben Mittelschicht.

In einem Topf verrührt mit solchen meist horizontalen Finanztransfers, wird die Finanzierung sozialer Dienstleistungen meist ausschließlich unter dem Aspekt des Aufwands wahrgenommen. Keinem Ökonomen würde es jedoch, in welchem Zusammenhang auch immer, einfallen, die Aufwandsseite zu kommentieren, ohne die Ertragsseite analysiert zu haben. »Sozi-

\section{"Die oft bemühte Formel, der Zweck Sozialer Arbeit sei es, sich selbst überflüssig zu machen, beschreibt einen fatalen Irrtum «}

dringendsten Bedürfnissen des Menschen, sich als bedeutsam zu erleben für andere. Und es gehört zu seinen vornehmsten Fähigkeiten, auf der Basis solcher Erfahrung anderen Menschen Bedeutung zu geben. Wird aber das Bedürfnis frustriert, dann erstirbt auch die alkosten"werden im Wirtschaftsteil einer jeden Zeitung thematisiert. Und ist dann auch einmal von "Sozialleistungen « die Rede, die man, nach dem ökonomischen Verständnis der Begriffe Kosten und Leistung, eigentlich einander gegenüberstellen müsste, dann sind 
in diesem Fall bloß noch einmal die Kosten gemeint.

Die oft bemühte Formel, der Zweck Sozialer Arbeit sei es, sich selbst überflüssig zu machen, beschreibt einen fatalen Irrtum. Diese Formel instrumentalisiert Soziale Arbeit und beschränkt sie auf das vermeintlich Notwendige. Aber Soziale Arbeit besteht nicht nur in der Wendung von Not. Sie besteht auch aus Beistand in der Not. Sie besteht zudem auch in der Stiftung von Gemeinschaft, in der Ermöglichung von Lebensfreude und Lebensqualität. Sie besteht schließlich auch aus der andauernden Assistenz angesichts von Einschränkungen, die durch solche Assistenz nicht verschwinden werden. Sie besteht zudem aus Bildung und Ausbildung sowie überhaupt aus der Freisetzung von Potentialen einschließlich der Ermächtigung, diese Potentiale ins Spiel zu bringen.
Wir brauchen Soziale Arbeit nicht nur um derer willen, für die sie geleistet wird. Wir brauchen sie auch um derer willen, die Soziale Arbeit leisten. Soziale Arbeit hilft einer doppelten Not auf, denn sie stiftet beiden, sowohl dem Klienten als auch dem professionellen Helfer, ein Gut, das immer knapper wird, wenn sich die Dinge auf dieser Welt mehr und mehr von allein bereitstellen: die Erfahrung nämlich, als Mensch bedeutsam $\mathrm{zu}$ sein für andere.

Soziale Arbeit zu ökonomisieren bedeutet vor diesem Hintergrund nichts anders, als ihr im Kontext der Arbeitsteilung einen nachvollziehbaren Wert und damit eine Bedeutung zuzuschreiben. Soziale Arbeit gehört deshalb auf den Markt. Sie muss rentabel werden. Nur dann entwickelt sie ihr Wachstumspotential. Unter den digitalisierten Verhältnissen, auf die wir zugehen, werden nicht die Ge- sellschaften reüssieren, die den Sektor sozialer Dienstleistungen kostenmäßig minimieren. Auch nicht jene, die diesen Sektor planwirtschaftlich verwalten. Reüssieren könnten aber Gesellschaften, die diesen Sektor als eine neue, volkswirtschaftlich relevante Branche begreifen und ihn deshalb so ordnen, dass den Nutzern die Kundenrolle zukommt. Auf dass diese, so weit als eben möglich, selbst entscheiden können, was gut für sie ist.

\section{Anmerkungen}

(1) Rosling, Hans u.a. Factfulness. Ten reasons we're wrong about the world - and why things are better than you think. London, 2018.

(2) Vgl. ausführlich:

Dopheide, Christian. Zur Digitalisierung des Sozialen. Baden Baden, 2017.

\title{
"Sehr empfehlenswert: Der erste Griff bei Fragen zum Mutterschutz, Elterngeld oder -zeit geht zu >dem Rancke‘."
}

RAin Birgit Scheibe, Sozialrecht aktuell 3/14, zur Vorauflage

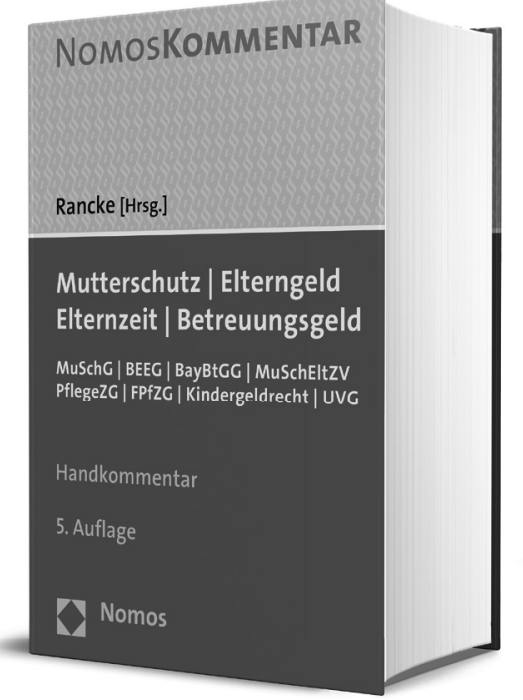

\author{
Mutterschutz | Elterngeld | Elternzeit | Betreuungsgeld \\ MuSchG | BEEG | BayBtGG | MuSchEltZV | \\ PflegeZG | FPfZG | Kindergeldrecht | UVG \\ Handkommentar \\ Herausgegeben von VRiLAG a.D. Dr. Friedbert Rancke \\ 5. Auflage 2018, 1.592 S., geb., 119,- $€$ \\ ISBN 978-3-8487-3401-6 \\ nomos-shop.de/28102
}

Der Kommentar gibt Antworten auf die wichtigen arbeits- und sozialrechtlichen Fragen der Elternschaft. Die juristischen Probleme der umfangreichen Änderungen des völlig neu strukturierten MuSchG werden detailgenau für die Praxis erläutert. Mitkommentiert ist das neue Bayerische Betreuungsgeldgesetz mit Referenzcharakter für zukünftige Landesgesetze auch in anderen Bundesländern.

Bestellen Sie jetzt telefonisch unter (+49)7221/2104-37.

Portofreie Buch-Bestellungen unter www.nomos-shop.de

Alle Preise inkl. Mehrwertsteuer

Nomos 